\title{
The Impact of the COVID-19 Pandemic on Bariatric Surgery: Results from a Worldwide Survey
}

\author{
loannis I. Lazaridis ${ }^{1}$ (D) Marko Kraljević $^{1} \cdot$ Romano Schneider $^{1}$ • Jennifer M. Klasen ${ }^{1} \cdot$ Dimitrios Schizas $^{2}$ • \\ Ralph Peterli ${ }^{1} \cdot$ Lilian Kow $^{3} \cdot$ Tarik Delko $^{1} \cdot$ for the Collaborators
}

Received: 12 May 2020 / Revised: 25 June 2020 / Accepted: 25 June 2020 / Published online: 11 July 2020

(C) Springer Science+Business Media, LLC, part of Springer Nature 2020

\begin{abstract}
Background The ongoing "coronavirus disease 19" (COVID-19) pandemic has had a strong effect on the delivery of surgical care worldwide. Elective surgeries have been canceled or delayed in order to reallocate resources to the treatment of COVID-19 patients. Currently, the impact of the COVID-19 pandemic on bariatric and metabolic surgical practice remains unclear.

Methods An internet-based online survey was performed among bariatric surgeons worldwide. The survey was sent to bariatric surgeons via the International Bariatric Club Facebook group and by electronic mail via the International Federation for the Surgery of Obesity and metabolic disorders (IFSO) secretariat to members of the associated national IFSO societies.

Results One hundred sixty-nine $(n=169)$ bariatric surgeons participated in the survey. The majority of the respondents postponed preoperative upper gastrointestinal tract endoscopies, appointments in the outpatient clinic and bariatric operations. Most surgeons performed video calls for follow-up appointments instead of meeting the patients in the outpatient clinics. Laparoscopy was still the preferred treatment for surgical emergencies, but a trend towards conservative treatment of acute appendicitis and acute cholecystitis was shown. Rapid preoperative COVID-19 testing availability was poor; therefore, routine screening of emergency bariatric cases was not widely provided. A wide variance occurred regarding precautions and personal protection equipment among the participants.

Conclusion The COVID-19 pandemic showed a strong impact on bariatric surgical practice regarding surgical and outpatient planning as well as personnel management. Coordinated effort from the national bariatric societies should focus on strict implementation of the current recommendations regarding precaution measures and personal protection equipment. Further studies should evaluate how this impact will evolve in the near future.
\end{abstract}

Keywords COVID-19 $\cdot$ Pandemic $\cdot$ Bariatric surgery $\cdot$ IFSO $\cdot$ Survey

\section{Introduction}

On March 11, 2020, the World Health Organization (WHO) declared "coronavirus disease 19" (COVID-19), which is caused by the severe acute respiratory syndrome coronavirus

Tarik Delko

tarik.delko@clarunis.ch

1 Clarunis, Department of Visceral Surgery, University Centre for Gastrointestinal and Liver Diseases, St. Clara Hospital and University Hospital Basel, Basel, Switzerland

2 First Department of Surgery, Laikon General Hospital, National and Kapodistrian University of Athens, Athens, Greece

3 Adelaide Bariatric Centre, Flinders Private Hospital, Bedford Park, Adelaide, South Australia, Australia
2 (SARS-CoV-2), a pandemic [1]. After its initial description in Wuhan City, Hubei province in China, cases of COVID-19 spread rapidly worldwide causing major global public health concerns. The COVID-19 pandemic has already shown to be a threat for the healthcare system of many countries. Governments have implemented social distancing measures and even nationwide lockdowns have been imposed in order to limit the spread of COVID- 19.

Elective surgeries have been canceled or delayed in order to increase intensive care unit (ICU) capacity and to reallocate resources, including personnel and equipment, to the treatment of COVID-19 patients in need of intensive care [2, 3]. Several strategies have been proposed among surgical disciplines regarding the triage of elective surgery and the management of surgical patients infected with SARS-CoV-2 [4, 5]. Outpatient visits have also been suspended or replaced by 
video consultations to avoid face-to-face contact between clinicians and patients $[6,7]$. Bariatric and metabolic surgery has been inevitably affected by the COVID-19 pandemic. However, the challenges faced by surgeons who perform bariatric surgery on their daily practice remain unclear. The objective of this survey is to understand the immediate international impact of the COVID-19 pandemic on healthcare systems, the role of bariatric and metabolic surgery and its change of practice, and the expected future implications in the surgical treatment of morbidly obese patients.

\section{Materials and Methods}

\section{Design and Subjects}

An internet-based online survey was conducted encompassing bariatric and metabolic surgeons globally via the International Bariatric Club Facebook group (Facebook Inc., California, USA) and directly by electronic mail via the International Federation for the Surgery of Obesity and metabolic disorders (IFSO) secretariat to members of the associated national IFSO societies. The survey included a questionnaire consisting of 42 items and could be completed in less than $5 \mathrm{~min}$. The questions were formulated by the authors based on the various controversies surrounding the global impact of COVID-19 on allocation of healthcare resources, protection issues, change of bariatric surgery practice, and the future implications for the surgical treatment of morbidly obese patients. The questionnaire was created using Google Forms (Google Inc., California, USA). Respondents were asked to confirm that they are attending surgeons or consultants practicing bariatric surgery. The email addresses of the participants were requested in order to complete a follow-up survey after 68 weeks aiming to evaluate how the current situation developed. The initial survey was open from April 1, 2020, until April 30, 2020.

\section{Outcome Measures}

The questionnaire was divided into five topics (baseline information, impact of the COVID-19 pandemic on daily bariatric practice, change of practice for morbidly obese patients during the COVID-19 pandemic, ICU capacity, precaution measures, see the "Appendix" section).

\section{Statistical Analysis}

Continuous data were presented as median with interquartile range (IQR). Categorical variables were summarized using frequencies and proportions. The chi-squared test was used and odds ratios (OR) with $95 \%$ confidence intervals $(\mathrm{CI})$ were reported as appropriate. The level of significance was set at $p<0.05$. All data analysis was performed using $\mathrm{R}$ version 3.5.3 [8].

\section{Results}

\section{Baseline Information of COVID-19 Impact on Healthcare Systems}

One hundred sixty-nine $(n=169)$ respondents participated in the survey. The median (IQR) age of the participants was 47 (41.3-55) years. The majority of the participants $(n=119,70$, 4\%) responded from the European IFSO Chapter. The baseline characteristics of the sample are reported in Table 1. One hundred fifty-five participants $(n=155,91.7 \%)$ indicated that wide-scale social distancing measures such as local and national lockdown had been implemented as a response to the COVID-19 pandemic in their country at the time they completed the survey. One hundred twenty-seven respondents $(n=127,75.1 \%)$ stated that patients with COVID-19 were being treated in their hospital at the time the survey was conducted, and one hundred five respondents $(n=105,62.1 \%)$ said they had COVID-19 patients receiving ICU treatment in their hospitals. Eleven participants $(n=11,6.5 \%)$ replied that COVID-19 infections had been diagnosed during hospitalization for elective bariatric procedures. Table 2 provides information about the situation regarding the COVID-19 pandemic in the hospitals of the participants during the study period as well as the response status to the pandemic at an institutional level.

\section{Impact of the COVID-19 Pandemic on Bariatric Surgery}

Forty-seven participants $(n=47,27.8 \%)$ stated that they considered changing the practice of preoperative work-up before bariatric surgery during the pandemic (Fig. 1). One hundred thirty-five $(n=135,80.4 \%)$, one hundred thirty-two $(n=132$, $78.1 \%)$, one hundred forty-seven $(n=147,87 \%)$, and one hundred forty-three $(n=143,84.6 \%)$ respondents postponed preoperative upper gastrointestinal tract (GI) endoscopies, appointments in the outpatient clinic (preoperative/ follow-up), primary and redo bariatric operations respectively. The majority of the responding bariatric surgeons $(78.7 \%, n=133)$ considered or already had performed follow-up via video call instead of visits in the outpatient clinic. Table 3 shows the respondents' expected delay period for elective bariatric cases caused by the precaution measures during the pandemic. Figure 2 illustrates the respondents' considerations about the impact of the COVID-19 pandemic on bariatric surgery practice in the future. $56.2 \%(n=95)$ of the participants believe that bariatric surgery may serve as a measure for risk reduction during respiratory infection pandemics such as the COVID-19 
Table 1 Baseline information of the study population

\begin{tabular}{|c|c|c|c|}
\hline & & $\begin{array}{l}\text { Median } \\
\text { (IQR) }\end{array}$ & $\begin{array}{l}\text { Total } \\
(\%)\end{array}$ \\
\hline Age (years) & & $47(41.3-55)$ & \\
\hline \multirow[t]{2}{*}{ Gender } & Male & & $138(81.7)$ \\
\hline & Female & & $31(18.3)$ \\
\hline \multirow[t]{6}{*}{ Region } & Europe & & $119(70.4)$ \\
\hline & Asia & & $21(12.4)$ \\
\hline & North America & & $20(11.8)$ \\
\hline & South America & & $5(3.0)$ \\
\hline & Australia & & $3(1.8)$ \\
\hline & Africa & & $1(0.1)$ \\
\hline \multirow[t]{3}{*}{ Hospital type } & Academic/Tertiary & & $52(30.8)$ \\
\hline & $\begin{array}{l}\text { Community/local } \\
\text { district }\end{array}$ & & $41(24.3)$ \\
\hline & Private clinic & & $76(45.0)$ \\
\hline \multirow[t]{5}{*}{ Number of hospital beds } & $<100$ & & $21(12.4)$ \\
\hline & $100-200$ & & $41(24.3)$ \\
\hline & $201-500$ & & $53(31.4)$ \\
\hline & $501-1000$ & & $40(23.7)$ \\
\hline & $>1000$ & & $14(8.3)$ \\
\hline \multirow{3}{*}{$\begin{array}{l}\text { Type of bariatric surgery } \\
\text { practice }\end{array}$} & Laparoscopic & & $142(84)$ \\
\hline & Robotic-assisted & & $3(1.8)$ \\
\hline & Both & & $24(14.2)$ \\
\hline \multirow{2}{*}{$\begin{array}{l}\text { Redo bariatric } \\
\text { procedures performed }\end{array}$} & Yes & & $156(92.3)$ \\
\hline & No & & $13(7.7)$ \\
\hline \multirow{2}{*}{$\begin{array}{l}\text { Emergency bariatric } \\
\text { procedures performed }\end{array}$} & Yes & & $148(87.6)$ \\
\hline & No & & $21(12.4)$ \\
\hline \multirow{2}{*}{$\begin{array}{l}\text { Preoperative upper GI } \\
\text { endoscopy performed }\end{array}$} & Yes & & $153(90.5)$ \\
\hline & No & & $16(9.5)$ \\
\hline
\end{tabular}

crisis, followed by $24.9 \%(n=42)$ and $18.9 \%(n=32)$ of the participants who responded "maybe" and "no" to this consideration (respectively).

\section{Change of Practice for Morbidly Obese/Post-Bariatric Patients During the COVID-19 Pandemic}

The vast majority of the participants $(88.7 \%, n=149)$ would not consider performing open surgery instead of laparoscopy for post- bariatric patients who require emergency surgery during the COVID-19 pandemic. In case of acute appendicitis in morbidly obese/post-bariatric patients, $59.3 \%(n=99)$ responded that they would still proceed with laparoscopic appendectomy. $34.7 \%(n=58)$ would choose conservative treatment instead of laparoscopic appendectomy in case of absent signs for peritonitis or perforation, whereas ten participants $(n=10,6.0 \%)$ consider change of practice to open appendectomy. Seventy-seven respondents $(n=77,45.8 \%$ ) would still proceed with laparoscopic cholecystectomy in case of an acute cholecystitis in a morbidly obese/post- bariatric patient, while seventy-eight participants $(n=78,46.4 \%)$ favor a conservative treatment trial with antibiotics. Only ten $(n=10,6 \%)$ and three $(n=3,1.8 \%)$ surgeons would replace laparoscopic cholecystectomy by cholecystostomy or open cholecystectomy respectively.

\section{ICU Capacity}

$39.6 \%(n=67)$ of the respondents routinely send patients with severe comorbidities to the ICU in the early postoperative period after bariatric surgery. The percentage of the respondents who routinely send patients with severe comorbidities to the ICU was significantly lower among surgeons working in community/local district hospitals than those working in academic/tertiary hospitals and in private clinics $(\mathrm{OR}=0.40$, 95\% CI: $0.18-0.89, p=0.027)$. The percentage of surgeons who send patients with severe comorbidities to the ICU did not differ significantly by number of hospital beds. Table 4 shows the association of bariatric surgeons sending patients with severe comorbidities to the ICU with hospital type and hospital size. $16.1 \%$ of the responders replied that patients have been sent directly to the ward during the COVID-19 pandemic, whereas thirty-eight surgeons $(n=38,22.8 \%)$ replied that they have considered sending patients directly to the ward instead of the ICU in case of a severe postoperative complication. Eighty-two $(n=82,48.8 \%)$ participants reported that they expect a shortage of the ICU beds in their institution during the COVID-19 pandemic, and fifty-two participants $(n=52,31.0 \%)$ stated that the ICU capacity had already reached its limit.

\section{Precaution Measures}

One hundred ten respondents $(n=110,65.1 \%)$ did not routinely test patients for COVID-19 before performing bariatric surgery. Thirty-four responders $(n=34,20.1 \%)$ answered that they currently test patients only if they show symptoms (Fig. 3). Rapid COVID-19 testing of emergency patients prior surgery was available in the hospitals of sixty surgeons $(n=60$, $35.7 \%$ ). Regarding expectations about shortage of protection material during the COVID-19 pandemic, $58 \%$ of the surgeons $(n=98)$ responded affirmatively and $31.4 \%(n=53)$ responded "maybe." One hundred six surgeons $(n=106$, $63.9 \%$ ) replied that they routinely adopted special precautions during bariatric surgery at the time of the survey. Figure 4 illustrates the different precaution measures in the operating theater. $80.5 \%$ of the respondents $(n=136)$ answered affirmatively about the self-isolation in case of present COVID-19 symptoms, while $7.7 \%(n=13)$ responded "maybe" and $11.8 \%(n=20)$ replied negatively. 
Table 2 The COVID-19 pandemic status at an institutional level during the study period as reported by the participants of the survey

\begin{tabular}{llr}
\hline & & Total (\%) \\
\hline $\begin{array}{l}\text { Patients treated with COVID-19 in } \\
\text { the ICU }\end{array}$ & Yes & $105(62.1)$ \\
& No & $59(34.9)$ \\
Number of total COVID-19 cases in & Unknown & $5(3 \%)$ \\
the respondents institutions & $10-20$ & $78(46.2)$ \\
& $21-50$ & $25(14.8)$ \\
& $51-100$ & $25(14.8)$ \\
& $>100$ & $20(11.8)$ \\
Availability of endoscopies & Elective and & $21(12.4)$ \\
& emergency & $41(24.3)$ \\
Protocols for selection of elective & Only emergency & $120(71)$ \\
surgical procedures & No availability & $8(4.7)$ \\
Protocols for protection of & Available & $146(86.4)$ \\
healthcare providers & Not available & $23(13.6)$ \\
Reduced surgical staff & Available & $163(97.0)$ \\
(due to absences/reduced work- & Not available & $5(3.0)$ \\
ing hours) & Yes & $140(82.8)$ \\
Shift of daily practice of & No & $29(17.2)$ \\
participants/members of their & Participation in & $88(52.1)$ \\
& general surgery on & \\
calls & Allocation in the ICU & $27(16)$ \\
& Reduced working & $104(61.5)$ \\
& hours & $30(17.8)$ \\
& Other & \\
& & \\
& &
\end{tabular}

\section{Discussion}

This survey of 169 bariatric surgeons representing all IFSO chapters worldwide is an immediate attempt to understand the various implications of the COVID-19 pandemic on the role and practice of bariatric and metabolic surgery. The main finding was a substantial change of the usual practice of bariatric surgeons such as preoperative work-up interventions (GI endoscopies), the postponement of planned primary and revision bariatric operations, and follow-up visits of post-bariatric patients. The majority of
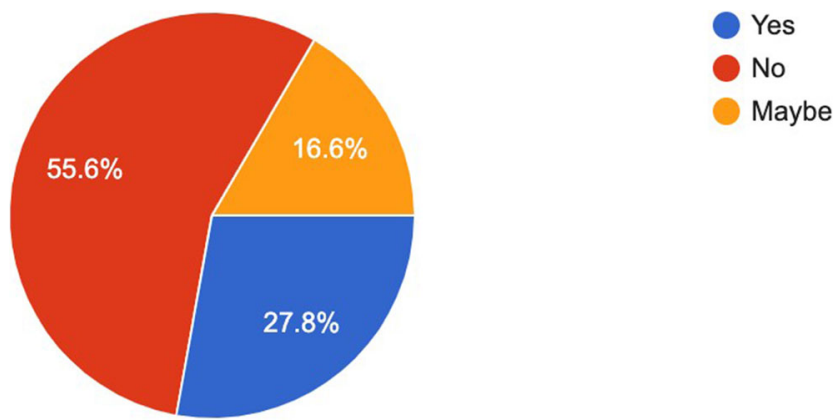

Fig. 1 To deal with the issues of the pandemic, have you considered changing your preoperative investigations before performing bariatric surgery? (169 responses) respondents changed their practice in accordance with international recommendations in regard to surgical planning, outpatient clinic, and management of personnel in order to increase healthcare capacity to a maximum and to protect staff and patients from COVID-19 transmission in hospitals in view of a possible worst case scenario as seen in Northern Italy.

In April 2020, IFSO published recommendations in regard to metabolic and bariatric surgical practice during the COVID19 pandemic [9]. In addition, the Society of American Gastrointestinal and Endoscopic Surgeons (SAGES) and The European Association for Endoscopic Surgeons (EAES) published joint recommendations for minimally invasive surgery during the COVID-19 pandemic. As a measure of resource allocation, the postponement of elective bariatric cases was strongly recommended. As a part of the surgical treatment prioritization, only emergency surgeries to treat complications of post-bariatric patients should be offered and conservative treatment should be considered first in case of suspected or confirmed COVID-19 cases [10]. Furthermore, the American College of Surgeons (ACS) provided guidance for the triage of non-emergent surgeries during the COVID-19 pandemic. They recommended that high acuity cases, including cancers and highly symptomatic patients, should be performed, while intermediate acuity surgeries should be postponed if possible. However, the ACS suggested a day-by-day assessment based on medical and logistical criteria should guide case triage [11]. Such recommendations are expected to strongly affect the time frame of elective bariatric surgical procedures. This is in line with the results of our survey, since $97.6 \%$ of the respondents expect delays of more than 4 weeks for postponed elective bariatric cases. Furthermore, according to the IFSO recommendations, follow-up visits at the outpatient clinics should be replaced by telephone or video visits [9]. The findings of our survey revealed that patient visits via telemedicine have already been widely adopted by the majority of bariatric surgeons.

Screening and protection of medical staff is a further controversy in the COVID-19 pandemic. IFSO recommendations about screening and protection during emergency surgery refer to suspected or confirmed COVID-19 cases. Symptom assessment, screening with chest computer tomography and confirmation by real-time reverse-transcriptase polymerase chain reaction, should be performed for all hospital admissions [9]. However, our survey revealed that routine screening

Table 3 Expectations of the participants about delays in planned bariatric operations

\begin{tabular}{lc}
\hline & $n(\%)$ \\
\hline$<4$ weeks & $4(2.4)$ \\
$4-8$ weeks & $58(34.3)$ \\
$8-12$ weeks & $73(43.2)$ \\
$>12$ weeks & $34(20.1)$ \\
\hline
\end{tabular}


Fig. 2 How do you consider that the COVID-19 pandemic will affect bariatric surgery in the future? (169 responses)

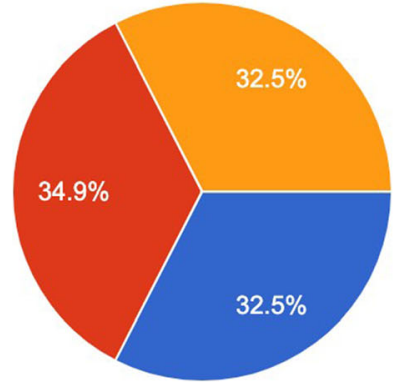

Increase in the volume of bariatric operations

Decrease in the volume of bariatric operations

No impact for COVID-19 of bariatric patients had not been implemented by most of the surgeons. In addition, a surprisingly high proportion of respondents $(64.3 \%)$ stated that rapid COVID-19 testing prior emergency surgery was not available at the time of the survey. Due to the high prevalence of pulmonary comorbidities, such as obstructive sleep apnea, obesity hypoventilation, and chronic obstructive pulmonary disease, bariatric patients are at risk for development of postoperative pulmonary complications. Therefore, they are vulnerable to a severe form of COVID-19 and to a subsequent complicated perioperative course with significant potential morbidity [12]. This signifies the importance of thorough screening for COVID-19 before bariatric surgery. Furthermore, negative pressure operating rooms, disposable surgical caps, medical protective masks (N95), disposable medical protective uniforms, disposable latex gloves, full-face respiratory protective devices, or powered air-purifying respirators are part of precaution measures in order to protect operating theater staff. In our study, we found that $63.9 \%$ of the participants routinely

Table 4 Association of number of respondents who send patients with severe comorbidities to the ICU after bariatric surgery with hospital type and hospital size

\begin{tabular}{lll}
\hline Factors & $\begin{array}{l}\text { Surgeons who send } \\
\text { patients with severe } \\
\text { comorbidities to the ICU } \\
(\% *)(n=67,39.6 \%)\end{array}$ & $\begin{array}{l}\text { Odds ratio } \\
(\% 95 \mathrm{CI})\end{array}$ \\
\end{tabular}

$\begin{array}{ll}\text { Hospital type } & \\ \text { Academic/tertiary } & 21(40.4) \\ \text { Community/local } & 10(24.4) \\ \text { district } & \\ \text { Private clinic } & 36(47.4)\end{array}$

$1.05(0.54-2.04) \quad 1.000$

$0.40(0.18-0.89) \quad 0.027$

$1.80(0.97-3.36) \quad 0.082$

Hospital size (number of hospital beds)

$\begin{array}{lrrr}<100 & 8(38.1) & 0.93(0.36-2.38) & 1.000 \\ 100-200 & 18(43.9) & 1.26(0.62-2.57) & 0.584 \\ 201-500 & 24(45.3) & 1.41(0.73-2.72) & 0.316 \\ 501-1000 & 13(32.5) & 0.67(0.32-1.41) & 0.356 \\ >1000 & 4(28.6) & 0.58(0.18-1.95) & 0.570\end{array}$

ICU intensive care unit

* The percentages refer to the total of surgeons who work in the same hospital type or in hospitals with the same size

$\% 95$ CI 95\% confidence intervals adopted special precaution measures during bariatric surgery. The expectancies of the respondents about shortage of protection material were worryingly high. The predominantly used precaution measure was eye protection, followed by double gloves and FFP3 face masks. Still, the frequencies of these types of personal protection equipment (PPE) revealed high variance. These findings demonstrate that the bariatric community has not yet been following a common pathway in terms of precaution measures to prevent harmful exposure to the virus. More coordinated effort should be made from the national bariatric societies towards strict implementation of the current recommendations in order to protect health care personnel from COVID-19 infections.

The matter of performing laparotomy instead of laparoscopy during the COVID-19 pandemic has been subject to debate, because of the potential risk of viral contamination via possible release of virus in the form of aerosol with $\mathrm{CO}_{2}$ during laparoscopy. Aerosolization of blood borne viruses through laparoscopy is a potential risk, which was shown in previous research [13]. SAGES/EAES recommendations still advise using minimally invasive surgery based on advantages such as reduced length of hospital stay, the lower risk of postoperative complications, and the option of using filters for aerosolized particles, which may be more difficult in open surgery [10]. Our study revealed accordance of the vast majority of respondents with the recommendation to use minimally invasive surgery. In case of post-bariatric emergency surgery, most respondents prefer the minimally invasive approach to an open approach whenever possible. Moreover, laparoscopy was still the preferred surgical option for acute appendicitis and acute cholecystitis. However, a significant percentage of surgeons (34.7\% and $46.4 \%$ respectively) would choose or had already chosen a conservative treatment with antibiotics over performing surgery whenever clinically feasible. This change of common practice in order to save resources for COVID-19 patients is another reflection of potential harm to patients suffering from other diseases during the pandemic.

Several reports have demonstrated a high mortality rate among patients with Type 2 Diabetes Mellitus (T2DM) and metabolic syndrome who contract COVID-19, while optimal metabolic control is suggested to help treating all patients with COVID-19. Moreover, comorbidities including T2DM and 
Fig. 3 Do you currently test patients for COVID-19 before performing bariatric surgery? (169 responses)

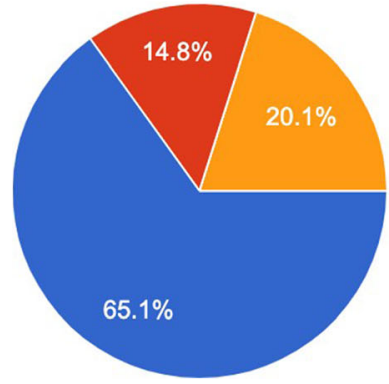

No

Yes- routinely

Yes- only if patients have symptoms hypertension have been associated with other coronavirus infections, including severe acute respiratory syndrome (SARS) and Middle East respiratory syndrome (MERS-CoV) [14]. The prevalence of obesity in patients with COVID-19 requiring admission to ICU and mechanical ventilation is highly compared with patients admitted to the ICU for other severe acute respiratory conditions [15]. Furthermore, a BMI of more than $30 \mathrm{~kg} / \mathrm{m} 2$ has been associated with an increased risk for hospital admissions and the need for ICU care in patients younger than 60 years [16]. Further data showed that younger patients admitted to the ICU are more likely to be obese [17]. These findings suggest that bariatric surgery for morbidly obese patients may play a key role in the prevention of developing severe disease caused by SARS-CoV-2, as well as by other coronavirus infections. Only $32.5 \%$ of the respondents of our survey suggested that the COVID-19 pandemic might lead to an increase of the volume of bariatric operations in the future, while $81.9 \%$ of the respondents agreed that bariatric surgery is or may be a means of risk reduction during respiratory infection pandemics. Further studies comparing the morbidity among morbidly obese patients and patients who have undergone bariatric operations are needed to provide such evidence.

Another important finding of this survey is the significant shift in the daily practice of bariatric surgeons worldwide. $61.5 \%$ of the respondents reported reduced working hours, while $52.1 \%$ of the respondents stated that they participated in general surgery on calls during the COVID-19 pandemic. These changes may have serious implications for the surgical practice, especially in regard to bariatric surgeons working in private practice settings. Due to the postponement of bariatric operations, preoperative investigations, and appointments, bariatric surgeons who work in private clinics may need to consider changing their practice mix by including more general surgery on calls. Moreover, surgeons maintaining private practice are in risk of shuttering their practice due to the loss of revenue caused by the reduced volume of bariatric operations.

This survey was conveyed when the COVID-19 pandemic was peaking in many regions globally, including Europe, North America, and many Asian countries. The strength of the survey is its global perspective representing a wide range of countries from all continents with a good distribution of answers from tertiary, community, and private clinics. To our knowledge, this is the first survey reporting these outcomes. However, there are several limitations to it. Although participation showed a global range, the majority of participants were European surgeons. The survey was sent out to IFSO members worldwide but a large number of bariatric surgeons have not participated in it. Moreover, due to the method of delivery of the survey, which included circulation on social media, we cannot provide a response rate. Since the survey was open for a period of 30 days, a reporting bias regarding the perspectives of the participants about the delays in planned bariatric procedures and the change of surgical practice may be possible. Nonetheless, we believe that our survey demonstrates the wide impact on bariatric surgery and the controversies surrounding surgical and outpatient management as well as staff protection and future perspectives of our field in view of the pandemic.
Fig. 4 What kind of precaution measures do you take? (141 responses)

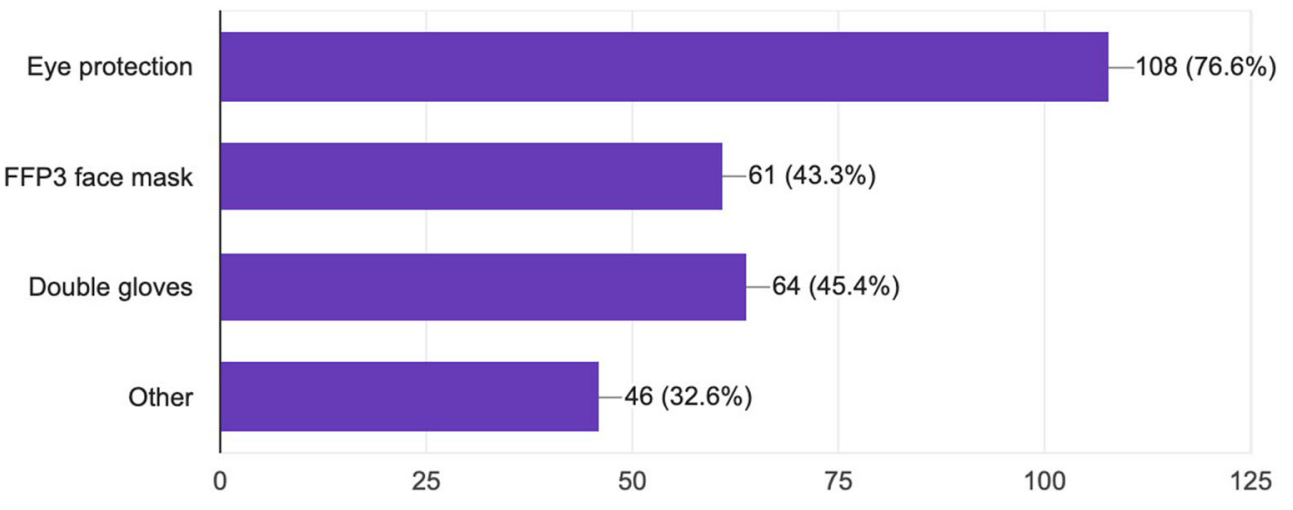




\section{Conclusion}

In view of a possible collapse of national healthcare systems, the COVID-19 pandemic calls upon all providers of medical services to offer direct or indirect support. Bariatric surgery departments and practices contributed immediately by postponing preoperative investigations and planned operations as well as offering telephone or video-based consultations for follow-up visits. Furthermore, we found a marked trend towards a conservative treatment of surgical emergencies among morbidly obese and post-bariatric patients, including acute appendicitis and acute cholecystitis, all of which may increase collateral damage to non-COVID-19-affected patients during the crisis. Patients' and healthcare providers' safety could be compromised due to the low routine preoperative screening availability for COVID-19 in emergency cases. Additionally, guidance in order to provide full PPE measures was not universally established. Future studies may reveal the role of bariatric surgery as a preventive measure, since obesity and the metabolic syndrome were found to be risk factors for worse outcomes in COVID-19 positive patients.

Acknowledgments The authors would like to thank Dr. Kazunori Kasama, Prof. Simon Msika, and Dr. Ronald Liem for their support for this survey.

The collaborators are: Patrick Noel, Rishi Singhal, Matthieu Girodet, Antoine Soprani, Olivier Brehant, David Moszkowicz, Xaviera Coueffe, Jean Sebastien Lanne, Ilan Rosenblum, Radu Moldovanu, Hannah Pflieger, Cyrille Kuperas, Pedro Martinez Duartez, Misha D. P. Luyer, Laurent Arnalsteen, Eric J. Hazebroek, Martin Sykora, Colleen Kennedy, Fabian Hauswirth, Riad Tchanderli-Braham, Sébastien Le Page, EvertJan G Boerma, Elias Chousleb, Barbara Langenhoff, Mohamad Hayssam ElFawal, Luca Paolino, Francesco Martini, Hubert Johanet, Thierry Barthes, Maen Halabi, Marco Del Prete, Felipe Eduardo Fiolo, Claire Blanchard, Ahmad Bashir, Monika Hagen, Ajjana Techagumpuch, Sebastien Dan, Jean-Marc Catheline, Constantin Georgeac, Liviu Paul Lefter, David Lechaux, Jeannette Widmer, Mirjam Anna Kaijser, Michal Orlowski, Peter François, Ann M. Rogers, Valentin Nitu, Stephane Servajean, Michael Fishman, Dana Mihaela Ignat, Jean Marie Molasoko, Marijn Takkenberg, Ennassih Amal, Mazen Charara, Chetan Parmar, Christophe Brévart, Eivind Andreas Warberg, Ludovic Marx, and Michel Vix.

Funding Information The authors did not receive any grant for this study.

\section{Compliance with Ethical Standards}

Conflict of Interest All authors declare that they have no conflict of interest related to this study.

Dr. Schneider reports grants from University Basel, grants from Department of Surgery, University Hospital Basel, grants from Freiwillige akademische Gesellschaft Basel, grants from Stiftung für chirurgische Forschung und Spitalmanagement, and grants from Gebauer Stiftung, outside the submitted work. Dr. Peterli reports grants from Johnson\&Johnson Switzerland, outside the submitted work.
Ethical Approval Statement The current study was a survey of expert surgeons and hence did not involve patients or animal subjects.

\section{Appendix}

\section{Baseline information}

1. Age

2. Gender

3. Country

4. Have wide-scale social distancing measures such as local and national lockdown been implemented as response to the COVID-19 pandemic in your country? (Yes/No)

5. Hospital type (private clinic/academic or tertiary hospital/community or local district)

6. Total number of hospital beds $(<100 / 100-200 / 201-$ 500/501-1000/> 1000)

7. Are there currently patients being treated with COVID19 in your hospital? (Yes/No/Unknown)

8. Are there currently patients being treated with COVID19 in the ICU of your hospital? (Yes/No/Unknown)

9. How many COVID-19 cases are currently treated in your hospital? $(<10 / 10-20 / 21-50 / 51-100 />100)$

10. What type of bariatric surgery do you perform? (Laparoscopic/Robotic-assisted/Both)

11. Do you perform redo bariatric surgery in your hospital? (Yes/No)

12. Do you perform emergency revisional surgery in your hospital? (Yes/No)

13. Is upper GI endoscopy performed as a preoperative investigation before bariatric surgery at your hospital? (Yes/No)

14. Are endoscopies available in your hospital during the COVID-19 pandemic? (Yes-both elective and emergency/Only emergency procedures/No)

15. Does your institution provide protocols for selection of surgical procedures during the COVID-19 pandemic? (Yes/No)

16. Does your institution provide protocols for protection of the health care providers during the COVID-19 pandemic? (Yes/No)

17. Is the staff of your surgical team currently reduced (because of absences/implementation of reduced working hours) due to the COVID-19 pandemic? (Yes/No)

18. Have you or any members of your team been subject to any shift of daily practice during the COVID-19 pandemic? (Participation in general surgery on calls/ Allocation in the ICU/Reduced working hours/other)

19. Has any of the bariatric patients been diagnosed with COVID-19 so far during an elective hospitalization in your hospital? (Yes/No) 


\section{Impact of COVID-19 pandemic on bariatric surgery}

20. To deal with the issues of the pandemic have you considered changing your preoperative investigations before performing bariatric surgery? (Yes/Maybe/No)

21. Have you postponed preoperative upper GI endoscopies during the COVID-19 pandemic? (Yes/No)

22. Have you postponed appointments (preoperative/followup) for bariatric patients in the outpatient clinic during the COVID-19 pandemic? (Yes/No)

23. Have you postponed planned primary bariatric operations during the COVID-19 pandemic? (Yes/No)

24. Have you postponed planned redo bariatric operations during the COVID-19 pandemic? (Yes/No)

25. Have you considered performing/ performed follow-up via video call (Skype, Facetime, etc.) instead of appointments in the outpatient clinic since the COVID-19 pandemic? (Yes/No)

26. With the current pandemic expecting to affect access to surgery, how long would you expect delays for performing postponed bariatric surgery? $(<4$ weeks/48 weeks $/ 8-12$ weeks/ $>12$ weeks)

27. How do you consider that the COVID-19 pandemic will affect bariatric surgery in the future? (Increase in the volume of bariatric operations/Decrease in the volume of bariatric operations/No impact)

28. Do you consider bariatric surgery as a means of risk reduction for morbidly obese patients during respiratory infection pandemics? (Yes/Maybe/No)

\section{Change of practice for morbidly obese patients during COVID-19 pandemic}

29. Have you considered performing/performed open emergency revisional surgery instead of laparoscopy for postbariatric surgery patients during the COVID-19 pandemic? (Yes/No)

30. In case of acute appendicitis in a morbidly obese/postbariatric surgery patient, have you considered/performed one of the following treatments instead of performing laparoscopic appendectomy during the COVID-19 pandemic? (No - proceed with laparoscopic appendectomy/ Conservative treatment with antibiotics/Open appendectomy)

31. In case of acute cholecystitis in a morbidly obese/postbariatric surgery patient, have you considered/ performed one of the following treatments instead of performing laparoscopic cholecystectomy during the COVID-19 pandemic? (No - proceed with laparoscopic cholecystectomy/Conservative treatment with antibiotics/Cholecystostomy/Open cholecystectomy)

\section{ICU capacity}

32. Do you send postoperatively patients with severe comorbidities to the ICU routinely? (Yes/No)

33. During the pandemic has there been any change in the postoperative destination of these patients? (Yes-patients are sent directly to the ward/No)

34. In case of a severe postoperative complication, have you considered sending/ have you sent patients directly to the ward instead of the ICU? (Yes/No)

35. Do you expect a shortage of ICU beds in your hospital during the COVID-19? (Yes/No/ICU beds already in shortage)

\section{Precaution measures}

36. Do you currently test patients for COVID-19 before performing bariatric surgery? (Yes-routinely/Yesonly if patients have symptoms/No)

37. Is rapid COVID-19 testing of emergency patients available prior surgery in your hospital? (Yes/No)

38. Do you expect a shortage of protection material during the COVID-19 pandemic? (Yes/Maybe/No)

39. Is personal protective equipment (PPE) available in your institution at all times during the COVID-19 pandemic? (Yes/No)

40. Do you and your team currently take special precaution measures during bariatric surgery? (Yes/No)

41. What kind of precaution measures do you take? (More than one choices possible) (eye protection/ FFP3 mask/ double gloves/other)

42. Do you or the members of your staff self- isolate/ consider self-isolating in case of having COVID-19 symptoms? (Yes/Maybe/No)

\section{References}

1. World Health Organization. Situat Rep. 2020;52.

2. Spinelli A, Pellino G. COVID-19 pandemic: perspectives on an unfolding crisis. BJS. 2020; https://doi.org/10.1002/bjs.11627.

3. Iacobucci G. Covid-19: all non-urgent elective surgery is suspended for at least three months in England. BMJ. 2020;368 https://doi.org/ 10.1136/bmj.m1106.

4. Zimmermann M, Nkenke E. Approaches to the management of patients in oral and maxillofacial surgery during COVID-19 pandemic. J Cranio-Maxillofacial Surg. 2020;48:521-6. https://doi. org/10.1016/J.JCMS.2020.03.011.

5. Tang D, Tou J, Wang J, et al. Prevention and control strategies for emergency, limited-term, and elective operations in pediatric surgery during the epidemic period of COVID-19. World J Pediatr Surg. 2020;3(1):e000122. https://doi.org/10.1136/wjps-2020000122. 
6. Lisi G, Campanelli M, Spoletini D, et al. The possible impact of COVID-19 on colorectal surgery in Italy. Color Dis. 2020;22:6412. https://doi.org/10.1111/codi.15054.

7. Greenhalgh T, Wherton J, Shaw S, et al. Video consultations for covid-19. BMJ. 2020;368:m998. https://doi.org/10.1136/bmj. m998.

8. R Core Team. R: A Langauge and environment for statistical computing. (Computer software). 2018.

9. Yang W, Wang C, Shikora S, et al. Recommendations for metabolic and bariatric surgery during the COVID-19 pandemic from IFSO. Obes Surg. 2020;30:2071-3. https://doi.org/10.1007/ s11695-020-04578-1.

10. Francis N, Dort J, Cho E, et al. SAGES and EAES recommendations for minimally invasive surgery during COVID-19 pandemic. Surg Endosc. 2020;34:2327-31. https://doi.org/10.1007/s00464020-07565-w.

11. American College of Surgeons. COVID-19: Guidance for Triage of Non-Emergent Surgical Procedures. https://www.facs.org/covid19/clinical-guidance/triage.

12. Aminian A, Kermansaravi M, Azizi S, et al. Bariatric surgical practice during the initial phase of COVID-19 outbreak [published online ahead of print, 2020 Apr 20]. Obes Surg. 2020:1-4. https://doi. org/10.1007/s11695-020-04617-x.
13. Schwarz L, Tuech JJ. Is the use of laparoscopy in a COVID-19 epidemic free of risk? Br J Surg. 2020;107:e188. https://doi.org/ 10.1002/bjs.11649.

14. Bornstein SR, Dalan R, Hopkins D, et al. Endocrine and metabolic link to coronavirus infection. Nat Rev Endocrinol. 2020;16:297-8. https://doi.org/10.1038/s41574-020-0353-9.

15. Simonnet A, Chetboun M, Poissy J, et al. High prevalence of obesity in severe acute respiratory syndrome coronavirus-2 (SARSCoV-2) requiring invasive mechanical ventilation. Obesity. 2020;28:1195-9. https://doi.org/10.1002/oby.22831.

16. Lighter J, Phillips M, Hochman S, et al. Obesity in patients younger than 60 years is a risk factor for Covid-19 hospital admission. Clin Infect Dis. 2020; https://doi.org/10.1093/cid/ciaa415.

17. Kass DA, Duggal P, Cingolani O. Obesity could shift severe COVID-19 disease to younger ages [published online ahead of print, 2020 May 4]. Lancet. 2020;S0140-6736(20):31024-2. https://doi.org/10.1016/S0140-6736(20)31024-2.

Publisher's Note Springer Nature remains neutral with regard to jurisdictional claims in published maps and institutional affiliations. 